\title{
Spinal Intramedullary Tuberculosis
}

\author{
Prithvi Varghese, MS, Mch ${ }^{1}$ Muhammed Jasim Abdul Jalal, MBBS, DNB \\ Julio Chacko Kandathil, MD, FRCR ${ }^{3}$ lona Leekha Mathew, MD ${ }^{4}$
}

${ }^{1}$ Department of Neurosurgery, VPS Lakeshore Hospital, Kochi,

Kerala, India

Address for correspondence Muhammed Jasim Abdul Jalal, MBBS, DNB, Department of Internal Medicine and Rheumatology, VPS

${ }^{2}$ Department of Internal Medicine and Rheumatology, VPS Lakeshore Lakeshore Hospital, NH-47 Bypass, Maradu, Nettoor P.O., Ernakulam, Hospital, Kochi, Kerala, India

${ }^{3}$ Department of Radiology, VPS Lakeshore Hospital, Kochi, Kochi 682040, Kerala, India (e-mail: jasimabduljalal@yahoo.com).

Kerala, India

${ }^{4}$ Department of Pathology, VPS Lakeshore Hospital, Kochi, Kerala, India

Surg J 2017;3:e53-e57.

\begin{abstract}
Keywords

- tuberculosis

- spinal

- intramedullary

- tuberculoma

Tuberculosis of the central nervous system accounts for approximately $1 \%$ of all cases of tuberculosis and $50 \%$ of these involve the spine. Intramedullary involvement is rare in tuberculosis. Clinical presentation of spinal intramedullary tuberculosis (SIMT) is similar to intramedullary spinal cord tumor. Here, we report the case of a 49-yearold female with dull aching pain of both upper limbs of 1-week duration. On examination, she had no motor deficits. All the deep tendon reflexes were normal. The plantar responses were flexor bilaterally. Cervical spine imaging favored intramedullary tumor. She had partial relief of symptoms with steroid treatment. Repeat imaging done 1 month later revealed mild interval enlargement of the intramedullary lesions and multiple enlarged mediastinal and hilar nodes. Endoscopic ultrasoundguided fine-needle aspiration cytology of mediastinal nodes was suggestive of granulomatous inflammation. Hence, SIMT was considered as the probable diagnosis. The patient was started on antituberculosis therapy.
\end{abstract}

According to the World Health Organization statistics for 2011 , out of the estimated global annual incidence of 8.7 million tuberculosis (TB) cases, 2.2 million were from India alone. TB of the central nervous system (CNS) accounts for approximately $1 \%$ of all cases of $\mathrm{TB}$ and $50 \%$ of these involve the spine. ${ }^{1,2}$ Spinal TB presents as various types of lesions.

Pott's disease of the spine (tuberculous spondylitis) is the most common (60\%), followed by arachnoiditis (20\%), meningitis (12\%), and intramedullary lesion (8\%). Meningitis is the most common form of spinal intradural TB. Intramedullary involvement is rare in TB and usually present in the form of radiculomyelitis, transverse myelitis, intraspinal granulomas, or thrombosis of anterior spinal artery. ${ }^{3}$ Clinical presentation of spinal intramedullary TB (SIMT) is similar to intramedullary spinal cord tumor. When diagnosed in time and managed appropriately, SIMT has good prognosis.

\section{Case Report}

A 49-year-old Indian female presented with dull aching pain of both upper limbs of 1-week duration. The patient also had episodes of difficulty in moving both upper limbs. There was no significant past illness. General examination was normal. The patient was afebrile with a pulse rate of 82 per minute and blood pressure of $140 / 80 \mathrm{~mm} \mathrm{Hg}$. She was conscious and oriented. PEARL [Pupils equal and reacting to light]. There were no motor deficits. All the deep tendon reflexes were received

October 28, 2016

accepted after revision

January 30, 2017
DOI http://dx.doi.org/

10.1055/s-0037-1599823. ISSN 2378-5128.
Copyright $\odot 2017$ by Thieme Medical Publishers, Inc., 333 Seventh Avenue, New York, NY 10001, USA Tel: +1(212) 584-4662.
License terms

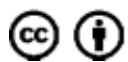


normal. The plantar responses were flexor bilaterally. All other systems were within normal limits.

Hemogram, liver function, renal function, and coagulation profile were all within normal limits. Magnetic resonance imaging (MRI) of cervical spine (-Figs. 1 and 2A, B) showed cord edema and swelling from C3 to C7 levels with two enhancing intramedullary cord lesions in anterior and right anterolateral aspect of cervical cord at C5-C6 level measuring $0.4 \times 0.4 \times 0.8 \mathrm{~cm}$ and $0.6 \times 0.7 \times 1.1 \mathrm{~cm}$, respectively. Cerebrospinal fluid (CSF) study was inconclusive (CSF was clear; CSF pressure: $120 \mathrm{~mm} \mathrm{H} \mathrm{H}_{2} \mathrm{O}$; CSF sugar: $48 \mathrm{mg} / \mathrm{dL}$; CSF protein: $21 \mathrm{mg} / \mathrm{dL} ; 5$ cells $/ \mathrm{mm}^{3}$ with occasional lymphocytes; and CSF culture was negative for pus cells, bacteria, and acid fast bacilli). At this stage, the possibility of an intramedullary tumor was considered. However, since the symptoms were rather acute in presentation, patient was treated with a course of steroids (intravenous methylprednisolone, $1 \mathrm{~g}$, once daily for 5 days).

Patient had partial relief of symptoms with steroid treatment. Repeat MRI done 1 month later revealed mild interval enlargement of the intramedullary lesions. However, the repeat MRI study also showed multiple enlarged mediastinal and hilar nodes (-Fig. 3A, B). Subsequent computed tomography of the thorax revealed extensive mediastinal and hilar adenopathy with no parenchymal lung lesion.

Endoscopic ultrasound-guided fine-needle aspiration cytology of mediastinal nodes showed several epithelial cell granulomas and lymphoid cells suggestive of granulomatous inflammation with suspicious small foci of necrosis

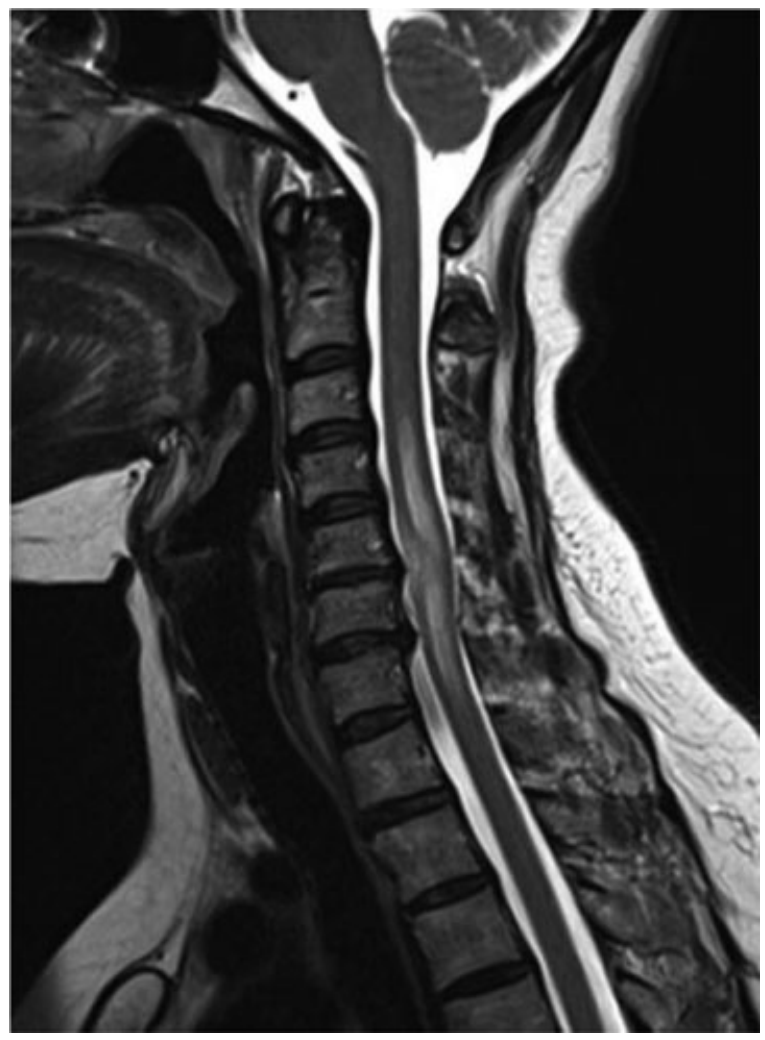

Fig. 1 Cervical spine sag T2-weighted image showing swollen cervical cord with T2-weighted high-signal edema extending from $\mathrm{C} 3$ to $C 7$ levels.

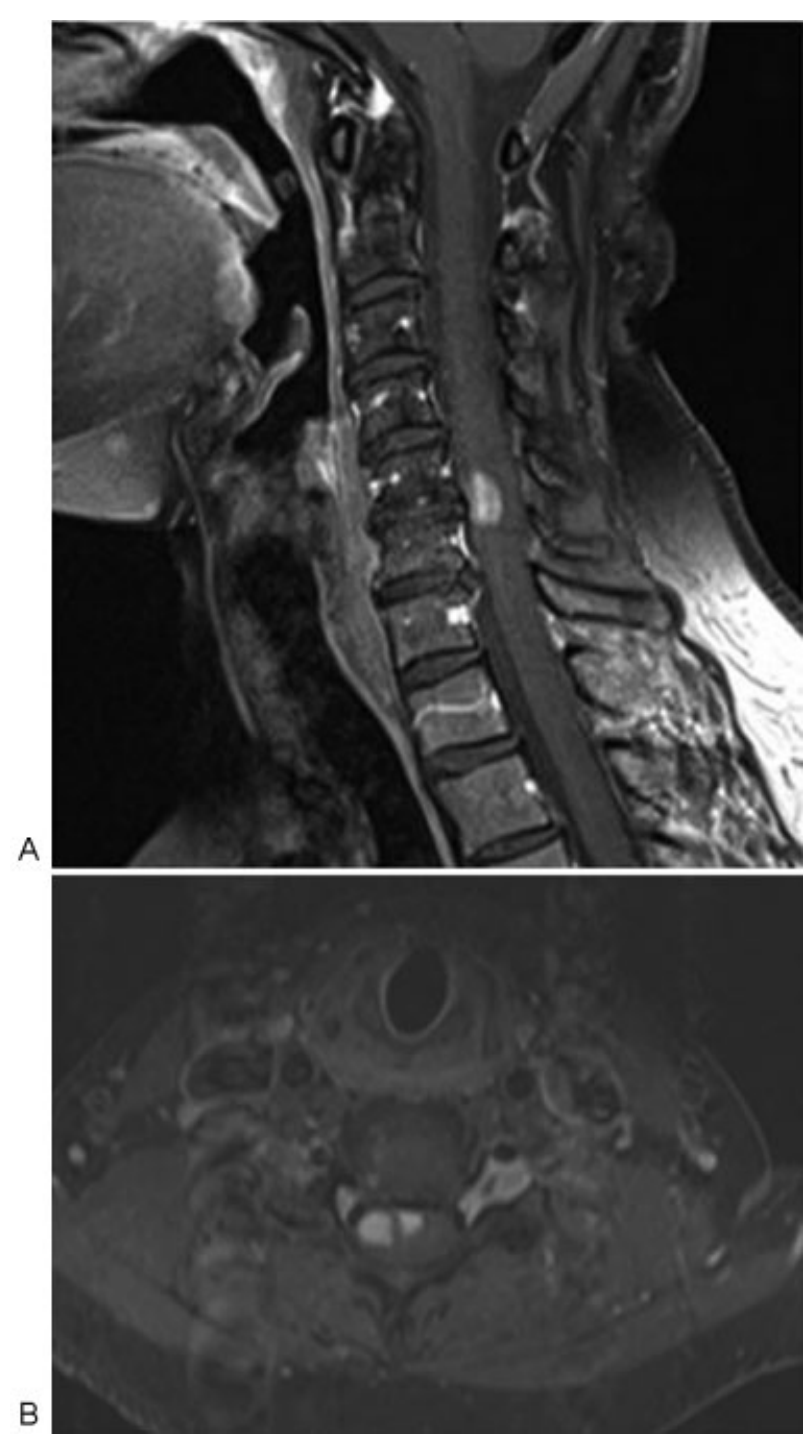

Fig. 2 (A) Cervical spine sagittal and (B) axial postcontrast T1weighted fat suppressed images showing enhancing intramedullary cord lesions at C5-C6 level situated in the anterior midline and right anterolateral aspect.

(-Fig. 4A, B). Hence, SIMT was considered as the probable diagnosis. The patient was started on anti-TB therapy with isoniazid, rifampicin, pyrazinamide, and ethambutol.

Repeat MRI of cervical spine (-Figs. 5 and $\mathbf{6 A}, \mathbf{B}$ ) after 3 months of ATT showed resolution of the cervical intramedullary lesions and oedema. There was minimal diffuse residual enhancement at the C5-C6 level. The patient completed 7 months of ATT with isoniazid, rifampicin, pyrazinamide, and ethambutol. Rifampicin and isoniazid was continued for another 2 months. She had total relief of her symptoms following the ATT. A repeat MRI was not done due to financial reasons. On follow up at 2 years following ATT, she is asymptomatic and has no neurological deficits.

\section{Discussion}

Spinal TB presents as various types of lesions. Pott's disease of the spine (tuberculous spondylitis) is the most common 


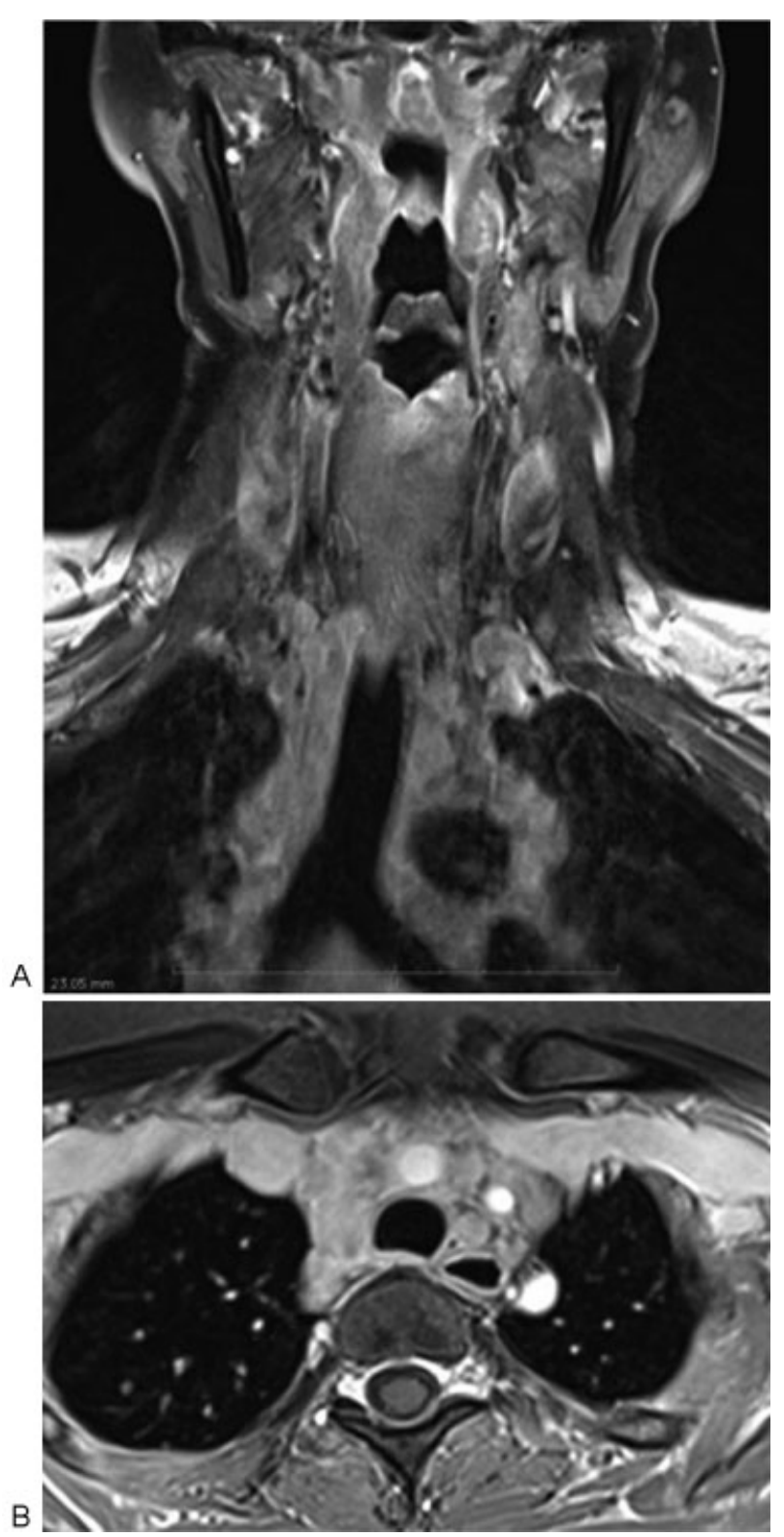

Fig. 3 (A) Coronal T1-weighted fat suppressed and (B) axial threedimensional volumetric interpolated breath-hold examination postcontrast images through mediastinum showing extensive mediastinal and hilar adenopathy.

(60\%), followed by arachnoiditis (20\%), meningitis (12\%), and intramedullary lesion (8\%). ${ }^{4}$ Dastur and Wadia described four major mechanisms that lead to spinal cord parenchymal involvement in $\mathrm{TB}^{5}$ :

- Edema of border zones of the cord probably secondary to venous impediment due to pressure associated with meningitis.

- Ischemic myelomalacia resulting from vasculitis or postthrombotic occlusion of meningeal vessels.

- Infarction of the cord from vascular occlusion.

- Formation of intramedullary tuberculomas with pericentral necrosis.

SIMT was first reported by Abercrombie in $1828 .{ }^{6}$ In 1960 , Arseni and Samitca ${ }^{7}$ reported 210 cases of CNS tuberculoma, of which only 9 were spinal masses. Linet $\mathrm{l}^{8,9}$ compiled 105 cases of intramedullary tuberculomas in 1960. In 17 of these cases, the diagnosis was made surgically, but 88 cases were discovered at postmortem examinations.

The types of lesions that result from discharge of the bacilli into the CSF depend on the virulence of the bacteria and the immune resistance of the host. A study of immunological parameters showed a relation between the development of tuberculous meningitis in children and significantly lower count of CD4 T-lymphocytes when compared with children who had pulmonary complex only. ${ }^{10}$

The brain lesion also originates from hematogenous spread from the lungs. When there is a sizeable inoculation with inadequate cell-mediated immunity, the parenchymal lesion may develop into a tuberculoma or a tuberculous abscess. $^{10}$

In Dastur's excellent review and summary of the tuberculoma series, 260 cases were in the brain and 6 were in the cord. Of the 74 tuberculous paraplegias in this group, 44 were extradural, 4 were subdural, 4 were combined subdural and extradural, and 6 were intramedullary. ${ }^{11}$

SIMT is mostly induced by hematogenous dissemination or CSF infection. However, in a few cases, it is caused by local spreading of spinal TB. It is important to rule out pulmonary TB or extrapulmonary TB in patients with SIMT. SIMT most commonly involves the thoracic spinal cord (55\%). ${ }^{12,13}$ SIMT is also reported in patients with human immunodeficiency virus, autoimmune disease, especially systemic lupus erythematosus, and patients undergoing immunosuppressive treatment due to liver transplantation. ${ }^{14}$ Patients frequently present with signs of spinal cord compression such as progressive lower limbs weakness, paresthesia, quadriplegia, paraplegia, and bladder and bowel dysfunction.

If diagnosed early, SIMT has good response to medical treatment. Anti-TB medications and a short course of injectable steroids offer an effective, inexpensive, safe, and feasible option for treating SIMT, especially in developing countries. ${ }^{15}$ Short-term steroids may be particularly helpful to reduce the perilesional edema. Usually, the conservative treatment is successful in achieving complete clinical neurologic recovery over a period of 1 to 2 years, which is also accompanied by resolution of the tuberculomas. ${ }^{16}$

Surgical intervention should be considered for cases showing progressive deficits in spite of adequate medical management and large lesions causing significant compression. With skilled microsurgical techniques, it is possible to safely excise the SIMT as these lesions are well circumscribed. MacDonnell et al have reported 65\% recovery after surgical treatment. ${ }^{16}$

Potential benefits of surgery were less kyphosis, immediate relief of compressed neural tissue, quicker relief of pain, higher percentage of bony fusion, quicker bony fusion, less relapse, earlier return to previous activities, and less bone loss. It may also prevent late neurologic problems due to kyphosis of the spine if fusion has not occurred.

Two types of surgical procedures are performed. One is debridement of the infected material. In this form of surgery, no attempt is made to stabilize the spine. The other 


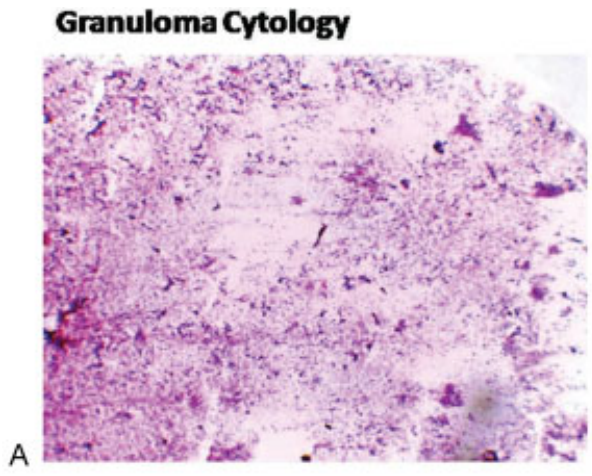

\section{Granuloma Clot}

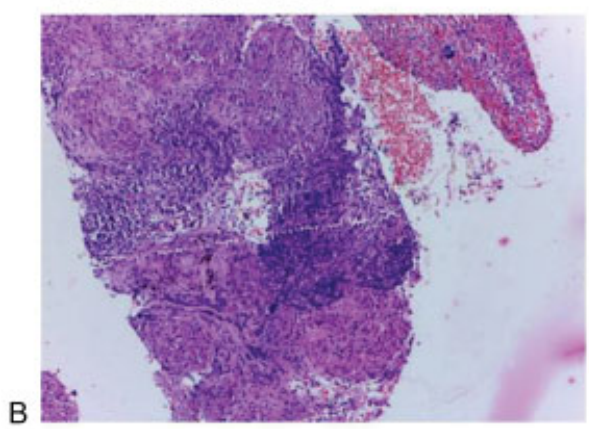

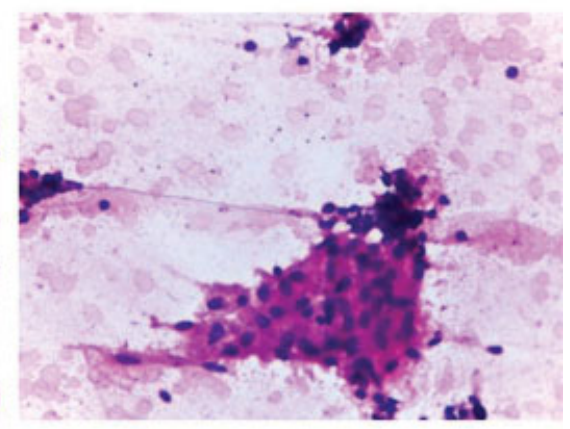

Granuloma Cytology-High Power

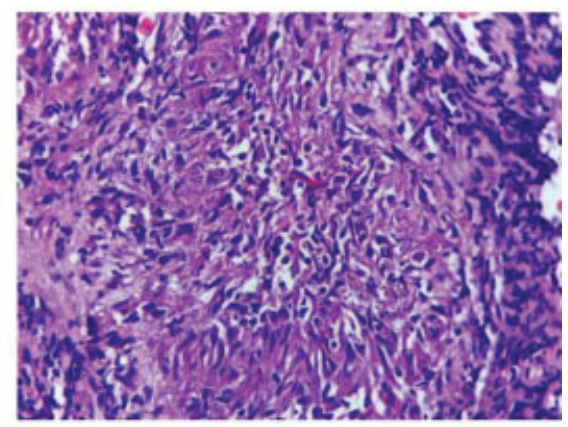

Granuloma Clot - High Power

Fig. 4 (A) Granuloma cytology. (B) Granuloma clot.

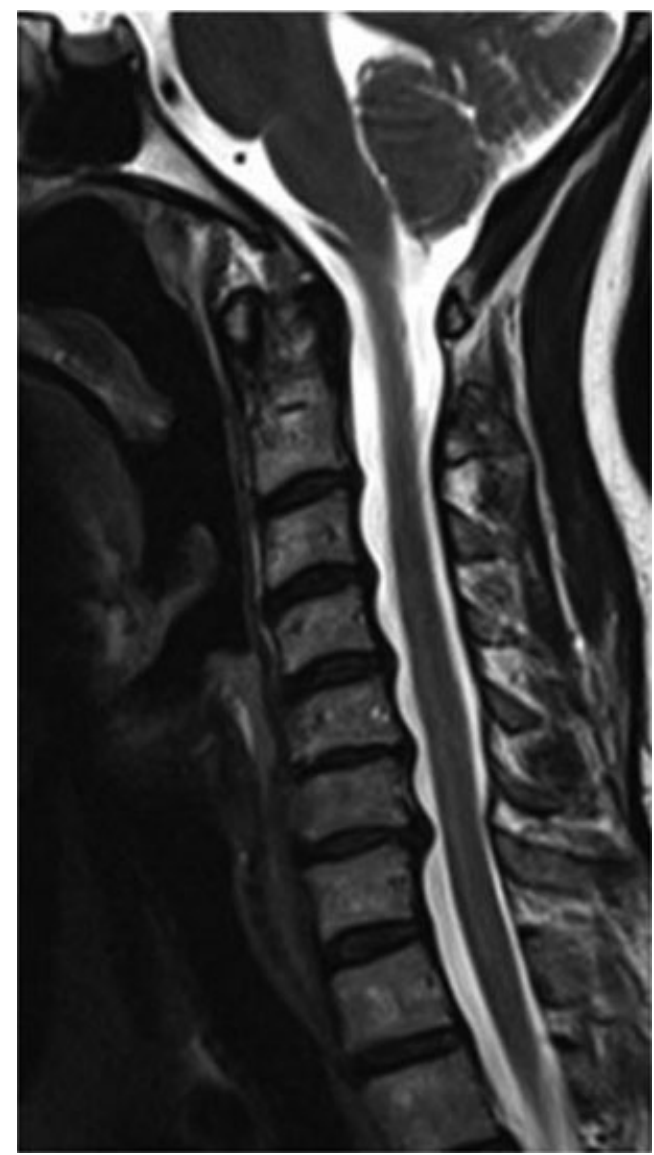

Fig. 5 Cervical spine sag T2-weighted image postantituberculous therapy showing resolution of cord edema. procedure is debridement with stabilization of the spine (spinal reconstruction). This is a more extensive procedure, and the reconstructions are performed with bone grafts. Stabilization may also be done using artificial materials such as steel, carbon fiber, or titanium.

In 2007, Park et al conducted a retrospective study and analyzed the treatment outcome in patients with spinal TB. Of 116 patients, 47 (35\%) had severe symptoms. Radical surgery was performed in 84 (62\%) patients. Twenty patients were treated with short-term chemotherapy, whereas 96 underwent long-term antituberculous treatment. At the end of chemotherapy, 94 patients had achieved a favorable status and 22 had an unfavorable one. Age and radical surgery were significantly related to a favorable outcome by logistic analysis. $^{17}$

The prognosis for spinal TB is improved by early diagnosis and rapid intervention. A high degree of clinical suspicion is required if patients present with chronic back pain, even in the absence of neurologic symptoms and signs. Medical treatment is generally effective. Surgical intervention is necessary in advanced cases with marked bony involvement, abscess formation, or paraplegia. Spinal TB affects young people; therefore, efforts should be made for its effective prevention. Controlling the spread of TB is only way available to prevent spinal TB.

Conflict of Interest

The authors have no conflicts of interest relevant to this article to disclose. 


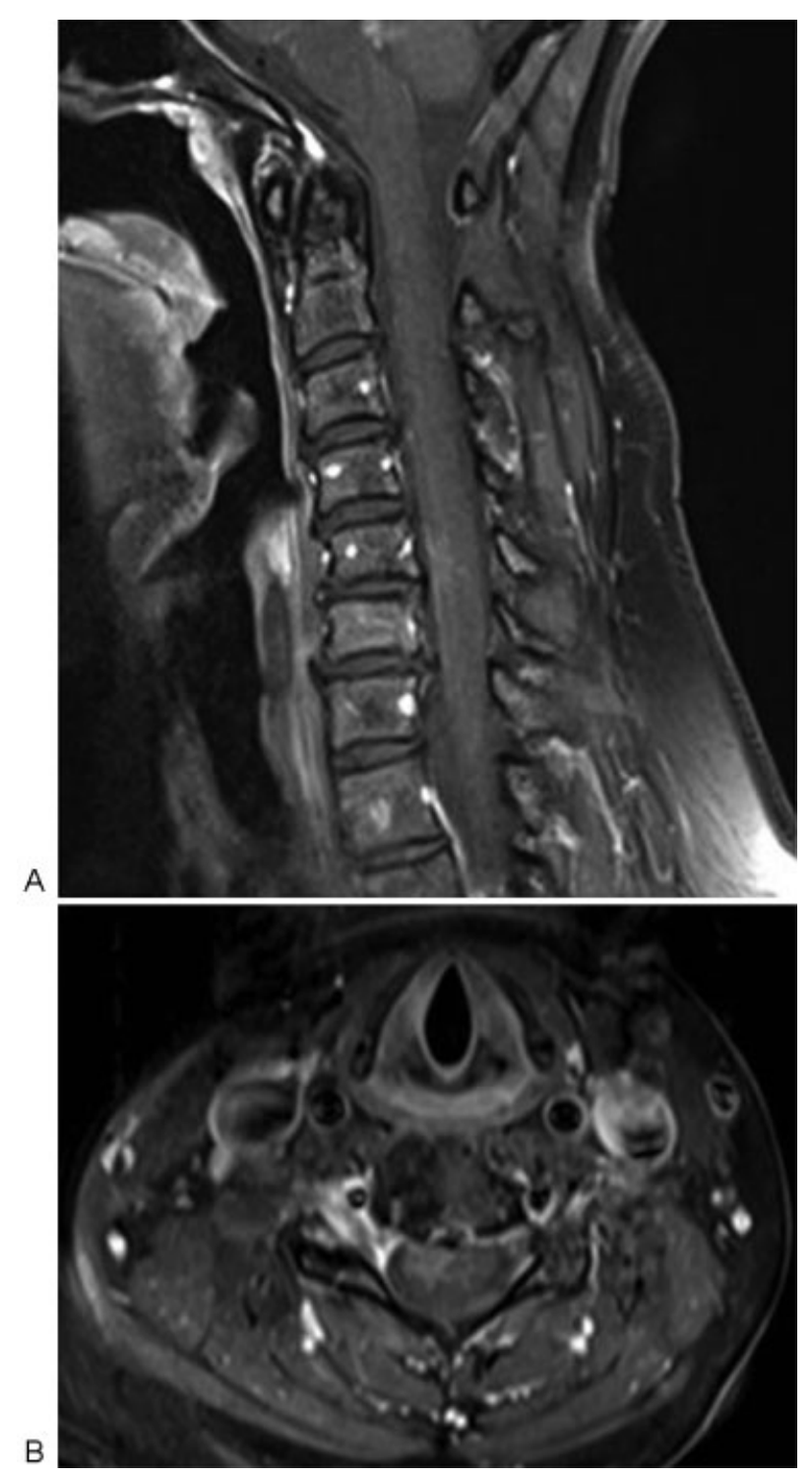

Fig. 6 (A) Cervical spine sagittal and (B) axial post contrast T1-weighted fat suppressed images postantituberculous therapy showing ill-defined residual enhancement at the site of C5-C6 level intramedullary lesions.

\section{Funding}

The authors have no financial relationships relevant to this article to disclose.

\section{References}

1 Cherian A, Thomas SV. Central nervous system tuberculosis. Afr Health Sci 2011;11(01):116-127

2 Jain AK, Dhammi IK. Tuberculosis of the spine: a review. Clin Orthop Relat Res 2007;460(460):39-49

3 Sohn S, Jin YJ, Kim KJ, Kim HJ. Long-term sequela of intradural extramedullary tuberculoma in the thoracic dorsal spinal cord: case report and review of the literature. Korean J Spine 2011;8(04):295-299

4 Muthukumar N, Venkatesh G, Senthilbabu S, Rajbaskar R. Surgery for intramedullary tuberculoma of the spinal cord: report of 2 cases. Surg Neurol 2006;66(01):69-74, discussion 74

5 Dastur D, Wadia NH. Spinal meningitides with radiculo-myelopathy. 2. Pathology and pathogenesis. J Neurol Sci 1969;8(02): 261-297

6 Abercrombie J. Pathological and Practical Researches on Disease of the Brain and the Spinal Cord. Edinburg: Waugh and Innes; 1828:371-372

7 Arseni C, Samitca DC. Intraspinal tuberculous granuloma. Brain 1960;83:285-292

8 Lin SK, Wu T, Wai YY. Intramedullary spinal tuberculomas during treatment of tuberculous meningitis. Clin Neurol Neurosurg 1994;96(01):71-78

9 Lin TH. Intramedullary tuberculoma of the spinal cord. J Neurosurg 1960;17:497-499

10 Chatterjee S. Brain tuberculomas, tubercular meningitis, and post-tubercular hydrocephalus in children. J Pediatr Neurosci 2011;6(Suppl 1):S96-S100

11 Dastur HM. A tuberculoma review with some personal experiences. II. Spinal cord and its coverings. Neurol India 1972;20(03): 127-131

12 Nussbaum ES, Rockswold GL, Bergman TA, Erickson DL, Seljeskog EL. Spinal tuberculosis: a diagnostic and management challenge. J Neurosurg 1995;83(02):243-247

13 MacDonnell AH, Baird RW, Bronze MS. Intramedullary tuberculomas of the spinal cord: case report and review. Rev Infect Dis 1990;12(03):432-439

14 Torii H, Takahashi T, Shimizu H, Watanabe M, Tominaga T. Intramedullary spinal tuberculoma-case report. Neurol Med Chir (Tokyo) 2004;44(05):266-268

15 Devi BI, Chandra S, Mongia S, Chandramouli BA, Sastry KV, Shankar SK. Spinal intramedullary tuberculoma and abscess: a rare cause of paraparesis. Neurol India 2002;50(04): 494-496

16 Ramdurg SR, Gupta DK, Suri A, Sharma BS, Mahapatra AK. Spinal intramedullary tuberculosis: a series of 15 cases. Clin Neurol Neurosurg 2009;111(02):115-118

17 Park DW, Sohn JW, Kim EH, et al. Outcome and management of spinal tuberculosis according to the severity of disease: a retrospective study of 137 adult patients at Korean teaching hospitals. Spine 2007;32(04):E130-E135 